\title{
Secondary B-cell lymphoma associated with the Epstein-Barr virus in chronic lymphocytic leukemia patients
}

\author{
Julie Morscio $^{1}$ - Emilie Bittoun ${ }^{1}$ - Nathalie Volders ${ }^{1} \cdot$ Eveline Lurquin $^{2}$. \\ Iwona Wlodarska $^{3}$ - Olivier Gheysens ${ }^{4} \cdot$ Peter Vandenberghe $^{3} \cdot$ Gregor Verhoef $^{5}$. \\ Philippe Demaerel $^{6}$ • Daan Dierickx ${ }^{5}$ - Xavier Sagaert ${ }^{1,2}$ - Ann Janssens ${ }^{5}$. \\ Thomas Tousseyn ${ }^{1,2}$
}

Received: 2 October 2015 / Accepted: 2 May 2016/Published online: 21 May 2016

(C) The Author(s) 2018, corrected publication May/2018

\begin{abstract}
Up to $10 \%$ of chronic lymphocytic leukemia (CLL) patients present with aggressive secondary B-cell lymphoma (most frequently diffuse large B-cell lymphoma, DLBCL) which may be clonally related to the CLL (i.e., Richter transformation, RT, $80 \%$ of the cases) or de novo (20\% of the cases). Several genetic lesions associated with RT have already been identified, but the potential role of the Epstein-Barr virus (EBV) has been largely overlooked.

In this study, we describe six CLL patients who developed a secondary EBV-positive $\left(\mathrm{EBV}^{+}\right)$B-cell lymphoma (five DLBCL, one Hodgkin lymphoma) and compare their clinicopathological characteristics to ten
\end{abstract}

The original version of this article was revised due to a retrospective Open Access order.

Electronic supplementary material The online version of this article (https://doi.org/10.1007/s12308-016-0273-8) contains supplementary material, which is available to authorized users.

Thomas Tousseyn

thomas.tousseyn@uzleuven.be

1 Department of Imaging and Pathology, Lab for Translational Cell and Tissue Research, KU Leuven, Leuven, Belgium

2 Department of Pathology, University Hospitals Leuven, Leuven, Belgium

3 Center of Human Genetics, University Hospitals Leuven, Leuven, Belgium

4 Department of Nuclear Medicine, University Hospitals Leuven, Leuven, Belgium

5 Hematology Department, University Hospitals Leuven, Leuven, Belgium

6 Department of Radiology, University Hospitals Leuven, Leuven, Belgium
CLL patients with EBV-negative $\left(\mathrm{EBV}^{-}\right)$secondary Bcell lymphomas (all DLBCL).

All 16 patients had a history of iatrogenic immunosuppression or chemotherapy. Eighty percent had received fludarabine as part of the CLL treatment. Most secondary lymphomas were clonally related to the previous CLL (3/4 $\mathrm{EBV}^{+}, 7 / 7 \mathrm{EBV}^{-}$cases tested). Notably $\mathrm{EBV}^{+} \mathrm{RT}$ was associated with a trend for older age at onset (median 72 vs. 63 years, $p$ value $>0.05$ ), longer interval between CLL and RT diagnosis (median 4.2 vs. 2.9 years, $p$ value $>0.05$ ), and shorter overall survival (median 4 vs. 10 months, $p$ value $>0.05$ ). These differences were not significant, probably due to small sample size. Immunohistochemical profiling suggested more frequent overexpression of TP53 and MYC in $\mathrm{EBV}^{-}$compared to $\mathrm{EBV}^{+}$secondary lymphoma.

Based on this small retrospective single center series, we hypothesize that $\mathrm{EBV}^{+} \mathrm{RT}$ may constitute a separate subgroup of RT. Larger series are required to validate this suggestion.

Keywords Epstein-Barr virus · Chronic lymphocytic leukemia $\cdot$ Richter transformation .

Immunosuppression-related lymphoproliferation .

Immunomodulatory agent-related lymphoproliferation .

Immunodeficiency-related lymphoma

\section{INTRODUCTION}

Up to $10 \%$ of patients with B-cell chronic lymphocytic leukemia (CLL), the most common type of adult leukemia, eventually develop an aggressive secondary lymphoma (most commonly diffuse large B-cell lymphoma, DLBCL) referred to as Richter transformation (RT) [1]. Although currently both clonally related and clonally unrelated secondary lymphomas 
are referred to as RT, recent (epi)genetic studies have demonstrated that these are actually distinct entities [2,3]. This is also apparent from the clinical setting as "true," i.e., clonally related cases of RT have a dismal prognosis compared to clonally unrelated DLBCL, which follows a disease course similar to de novo DLBCL [4].

In recent years, a number of risk factors for RT development have been defined including loss/mutation of TP53 [5, 6], NOTCH1 mutations [7], and the presence of the unmutated immunoglobulin heavy variable gene IGHV4-39, which is frequently part of the B-cell receptor in the DLBCL variant of RT [8]. These observations may be clinically useful for prompt recognition of RT as the outcome is influenced by the extent of disease [9, 10]. Regarding RT pathogenesis, several genetic lesions have been identified [11]. Notably, a few studies have addressed the potential role of the Epstein-Barr virus (EBV), an oncogenic human herpesvirus, in this process. However, because EBV is only occasionally detected in cases of RT [12,13], its role as a driving factor is unclear. In vitro studies have shown that CLL cells are not easily infected by EBV and that cytokine signaling in the microenvironment may be crucial for rendering CLL cells susceptible to EBV infection [14]. Rossi et al. suggested that EBV-positive lymphoma in the context of CLL represents a novel type of immunodeficiency-related lymphoma that may develop following T-cell depletion or chemotherapy [9]. Following an intriguing case of EBV-related large B-cell transformation in the brain of a CLL patient, we reviewed all cases of secondary B-cell lymphoma in CLL patients available in our archive. This series was screened for the presence of EBV in an attempt to determine the clinicopathologic characteristics and possible prognostic implications of the involvement of EBV. Based on our results, we suggest that EBV-positive $\left(\mathrm{EBV}^{+}\right) \mathrm{RT}$ may represent a distinct subgroup of RT. We therefore encourage monitoring of EBV titers in newly diagnosed CLL and systematic screening for EBV in RT, particularly in elderly patients, to validate this hypothesis in a larger series of patients.

\section{Materials and methods}

\section{Patient selection, histopathology, and statistics}

In this retrospective single-center study, the clinicopathologic characteristics of 16 secondary B-cell lymphoma cases (retrieved from the archives of the University Hospitals Leuven) are described. All slides were reviewed by an expert hematopathologist (TT), and diagnosis was made according to the 2008 WHO criteria [15]. Clinical information was retrieved for all cases from the medical records. The Ethical Committee of the University Hospitals of Leuven approved this study (S-55498) which was conducted according to the Declaration of Helsinki.
EBER (EBV-encoded RNA) in situ hybridization was performed using a 30-mer digoxigenin-labeled oligonucleotide probe (Research Genetics, Huntsville, AL), according to the manufacturer's instructions. To check the RNA integrity, a control poly-A probe (Ventana Roche, Arizona, USA) was applied in parallel on all cases. A proven EBV-driven lymphoma was used as a positive control. Cases were defined as EBV-positive when the majority of viable tumor cells were EBER-positive.

Two antibody panels were applied to characterize the tumor (CD20, CD5, CD23, CD10, BCL-6, MUM-1, BCL2, Mib1, CyclinD1, TP53, MYC) and the viral protein expression pattern (LMP1, EBNA2, ZEBRA). All stainings were performed automatically according to manufacturers' protocol.

Statistical analysis was performed using Statistica 7.0 (StatSoft) and consisted of univariate Kaplan-Meier analysis (log-rank and Wilcoxon test) and the Mann-Whitney nonparametric $t$ test.

\section{Immunoglobulin (Ig) PCR and somatic hypermutation assay}

$I G H$ and $I G K$ rearrangements of CLL and secondary DLBCL were studied by multiplex PCR with BIOMED-2 primers using a BIOMED-2 PCR based protocol [16]. The somatic hypermutation assay was performed on secondary DLBCL by standard methods from the clinical laboratory. The mutational status was ultimately determined using the IMGT database [17].

\section{RESULTS}

\section{Case reports of EBV-positive Richter transformation}

\section{Case 1}

In November 2000, a 72-year-old male patient presented with leukocytosis, compatible with a CLL, without lymphadenopathy. Cytogenetics showed del(11)(q22q24) resulting in loss of ATM. The patient received chlorambucil for 10 months. After 1 year, chlorambucil was restarted for increasing leukocytosis and lymphadenopathies. Because of disease progression in the bone marrow (91\% CLL cells; no transformation) and persistent thrombocytopenia, therapy was switched to fludarabine in April 2004. In December 2004 (after five administrations of fludarabine), the patient was admitted to the hospital because of general weakness, dizziness, and persistent cough. He had recently developed bilaterally enlarged cervical lymph nodes. EBV DNA had risen from undetectable levels (at time of diagnosis of CLL) to 16,100 copies $/ \mathrm{ml}$ 2 weeks before biopsy and to $44,497 \mathrm{copies} / \mathrm{ml}$ at the time 
of biopsy. Histopathology revealed an EBER-positive nongerminal center B-cell DLBCL, with central necrosis. Cytogenetic analysis revealed evolution of the del(11q)-positive karyotype. The patient passed away shortly after one administration of rituximab, cyclophosphamide, doxorubicin, vincristine, and prednisolone (R-CHOP) due to the development of chemotherapy-induced pancytopenia complicated with neutropenic fever and sepsis.

\section{Case 2}

In October 2000, a 70-year-old male patient was diagnosed with CLL, but no treatment was installed. In May 2001, he developed an episode of CLL-associated idiopathic thrombocytopenic purpura, which responded well to steroids. In December 2002, chlorambucil was started because of increasing doubling time of leukocytes and development of cervical lymphadenopathies. In February 2003, fludarabine was initiated but stopped after two cycles because of persistent thrombocytopenia, and administration of steroids was restarted. In May 2003, the patient received four cycles of rituximab. Steroid administration could not be completely discontinued because of persistent thrombocytopenia. In January 2005, chlorambucil was restarted because of increasing lymphadenopathies. After four cycles, the patient developed a necrotizing suppurative right inguinal lymphadenopathy, without evidence for CLL localization. A CT scan showed atrophic changes and periventricular vascular leukomalacia in the brain. Retroperitoneal lymph nodes were significantly enlarged, with necrotizing center. A PET scan showed multiple moderately active mediastinal, mesogastric, retroperitoneal lymphadenopathies. Histopathology of a retroperitoneal mass revealed a CLL in transformation to a DLBCL, with central necrosis. Cytogenetics showed a complex karyotype including the del(13)(q14q22), characteristic for CLL. R-CHOP with a $50 \%$ dose reduction of doxorubicin was started. Rituximab was stopped after one administration due to a severe hypotension. One week after CHOP, during the neutropenic phase, the patient passed away due to sepsis.

\section{Case 3}

In 2004, a 57-year-old female patient was diagnosed with CLL and treated with chlorambucil and fludarabine. She was lost to follow-up for 2 years during which she received four cycles of R-CHOP for which the indication is unclear. RCHOP was discontinued and she remained in complete remission for 1.5 years. In 2009, she relapsed with CLL; cytogenetics of peripheral blood showed loss of $13 q 14$ in the tumor cells. In December 2010, alemtuzumab therapy was started. This was accompanied by a gradual rise in the EBV DNA titer from undetectable levels to weakly positive. In April 2011, alemtuzumab was stopped because of severe pancytopenia.
At that point, she was in partial remission: she still had some small lymphadenopathies in the inguinal and iliacal regions and at the liver hilus. In August 2011, the patient developed a thrombosis of the large saphenic vein due to compression by enlarged inguinal lymph nodes. Biopsy showed an EBVpositive DLBCL of non-germinal center B-cell origin clonally unrelated to the CLL. At that time, the EBV DNA titer peaked (1821 copies/ml). Following five cycles of rituximab monotherapy, the EBV DNA titer dropped to $<500$ copies/ml and the patient went in partial remission. Three and a half years later, she is in relatively good condition and she receives monthly substitution of IV immunoglobulins. Since followup after completion of rituximab therapy, the EBV titers gradually rose between December 2011 (3422 copies/ml) and July 2013 to 4135 copies $/ \mathrm{ml}$. Thus far, the patient is not developing new lymphadenopathies.

\section{Case 4}

In February 2000, a 62-year-old male patient with a history of prostate cancer (diagnosed in 1995) treated with prostatectomy, radiotherapy, and hormone therapy (Zoladex) was diagnosed with a common B-cell acute lymphoid leukemia (BALL). Cytogenetics revealed a $t(1 ; 19)(q 23 ; p 13)$ resulting in the E2A-PBX1 fusion. Complete remission was reached after two cycles of chemotherapy (vincristine, daunorubicin, methylprednisolone, and asparaginase) and three cycles of intrathecal methotrexate. In July 2011, further investigations were performed because of chronic fatigue and 4-kg weight loss over a period of 6 months. There was no morphological or molecular evidence for relapse of B-ALL (E2A-PBX1-negative by RTqPCR). PET scan showed multiple faintly fluorodeoxyglucoseavid, slightly enlarged lymph nodes (bilateral axillar, retroperitoneal, iliacal, liver hilus, hepatogastric, mesenterial, retrocrural). A diagnosis of CLL was made and a watchful waiting approach was installed. All clinical and radiological parameters were stable for the next year.

In October 2012, the patient was admitted to the hospital with sudden gait instability and two episodes of falling over the last 2 weeks. He complained of a tingling sensation in the right arm and leg. Over several months, he became progressively depressed and lost $4 \mathrm{~kg}$. Clinical examination showed unstable gait, a tendency to fall to the left and a left central facialis paresis, but he had no headache, vertigo, loss of consciousness, fever, night sweats, or other associated symptoms. CT and MRI of the brain demonstrated a polylobular contrastcaptating tumor in the right parietotemporal area with perilesional edema and mass effect, highly suspicious for a glioblastoma (Supplementary Fig. 1). Histopathological examination of the lesion, however, showed no evidence for a glioblastoma but revealed a dense lymphoid infiltrate with two components. The leptomeninges and superficial cortex showed a predominantly perivascular lymphocytic infiltrate, 


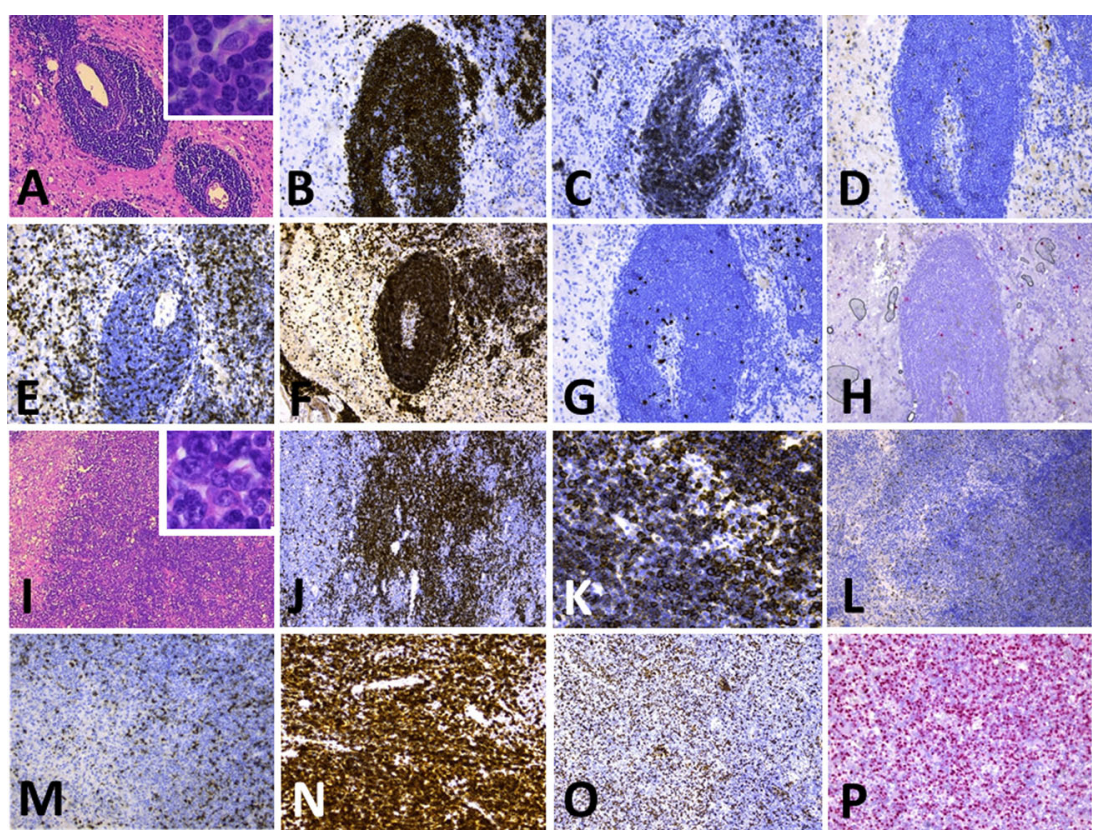

Fig. 1 Histopathology of the brain biopsy of case 4 containing both CLL and Richter transformed CLL. (a)-(h) shows the presence of CLL in the brain; (i)-(p) shows the large cell Richter transformed diffuse large B-cell lymphoma (DLBCL) in the brain. a, i HE of perivascularly localized CLL (a) and the corresponding DLBCL (i). The insets show the tumor cells at larger magnification $(\times 400)$. CLL and DLBCL were positive for PAX5 (b, j); however, CD20 was more weakly expressed in CLL (c) compared

composed of small round lymphocytes with condensed chromatin and scant cytoplasm. Mitotic activity was virtually absent. The small cell component expressed the mature B-cell markers CD20, CD79a, and PAX5, and aberrant CD5 and CD23, but no CD10, CyclinD1, nor TdT. The deeper cortex showed a large blastoid lymphoid proliferation with extensive necrosis and increased mitotic and apoptotic activity. These cells had the same immunophenotype as the small-cell component, except for the presence of EBER in the large blastoid population and to a minor extent in the small cells. Laser microdissection was performed and DNA was extracted from the CLL and large-cell component. PCR confirmed the presence of a monoclonal B-cell population, identical to the CLL clone found in 2011, but different from the B-ALL clone in 2000. Diagnosis of a secondary central nervous system lymphoma with histological features of an EBV-induced RT of CLL to DLBCL was made (Fig. 1, Supplementary Table 2). The right parietotemporal lobe was irradiated $(15 \times 2$ Gy), but the patient died 3 months later.

\section{Case 5}

In 1997, a 65-year-old male patient was diagnosed with CLL, with prognostically favorable (mutated $\mathrm{IgVH}$ gene status; CD38 negative, del(13)(q13q21)) and unfavorable del(17p) characteristics. From 2001 until 2003, he was treated with chlorambucil. In April 2006, chlorambucil was restarted to DLBCL (k). Both components were negative for cyclin D1 (d, l). CD3 staining revealed the presence of scattered T cells $(\mathbf{e}, \mathbf{m})$; however, staining for the T-cell marker CD5 demonstrated strong expression in both CLL and DLBCL $(\mathbf{f}, \mathbf{n})$. Proliferation was limited in CLL (as evidenced by Mib1 staining, g) while the vast majority of DLBCL cells were actively proliferating $(\mathbf{g})$. Notably, EBER ISH revealed some sparse positive small cells in CLL (h) and diffuse positivity in the large cells (p)

because of recurrent lymphocytosis, mild anemia, and thrombocytopenia. Because of intolerance, chlorambucil was switched to fludarabine in June 2006. Because of persistent hepatosplenomegaly, fludarabine was discontinued after four cycles and alemtuzumab was started in October 2006. The patient received 12 cycles, but 2 weeks after the last injection, he presented with fever, diffuse abdominal pain, anorexia, but no B-symptoms. Bone marrow biopsy showed residual lymphoma without morphological evidence of transformation, but with cytogenetic evidence for clonal evolution. Inguinal and multiple retroperitoneal as well as mesenteric lymphadenopathies were present. Both spleen and liver were enlarged, and the latter showed multiple hyporeflective nodules at ultrasound. Liver function progressively decreased with concomitant abdominal ascites fluid accumulation. A liver biopsy revealed an $\mathrm{EBV}^{+}$diffuse large B-cell lymphoma with postgerminal center phenotype. The patient was transferred to intensive care where his situation deteriorated. Two days later, the patient died due to disease progression.

\section{Case 6}

In 2003, a 58-year-old female patient with a history of lobular breast carcinoma was diagnosed with CLL. After 5 years of watchful waiting, she was included in the experimental arm of the Lucid trial (lumiliximab, fludarabine, cyclophosphamide, rituximab). After completion of the fourth cycle of rituximab 
in November 2009, she developed subungual bleedings and lymphadenopathies. After initial watchful waiting and rising EBV titers (from 9389 copies $/ \mathrm{ml}$ in November 2009 to 42, 671 copies/ml in February 2010), she developed B-symptoms. A bendamustine and rituximab regimen was initiated in March 2010 as an EBV-driven lymphoma was suspected. CT showed disease progression with multiple supra- and infradiaphragmatic lymphadenopathies and splenomegaly. Lymph node excision showed the presence of an $\mathrm{EBV}^{+}$lymphoproliferation compatible with mixed cellularity type classical Hodgkin lymphoma (cHL), for which ABVD (doxorubicin, bleomycin, vinblastine, dacarbazine) was started. The lymphoproliferation was monoclonal, but the clonal relationship with the CLL was unclear due to incomplete Ig PCR results. Huge Reed-Sternberg cells showed JAK2 amplification, recurrent in cHL [18]. Due to disease progression, the patient died in November 2010.

\section{Summary of clinical and pathological results}

The clinical and pathological characteristics of this series of 16 secondary B-cell lymphomas are summarized in Tables 1 and 2 . CLL was diagnosed at a median age of 65 years (range 3876 years). Aberrations characteristic for CLL (del 11q, 13q, 17 p, trisomy 12) were found in all nine analyzed cases.
Overall, $63 \%$ of the secondary B-cell lymphomas occurred in male patients, at a median age of 70 years and following a median interval of 4 years after diagnosis of CLL. All were DLBCL of non-germinal center B-cell origin except for one case of classic Hodgkin lymphoma (no. 6). EBER was detected in $38 \%(6 / 16)$ and was associated with higher MUM1 expression $\left(100 \%\right.$ of $\mathrm{EBV}^{+}$cases were positive compared to $67 \%$ of $\mathrm{EBV}^{-}$cases). Compared to the $\mathrm{EBV}^{-}$cases, we observed a trend that $\mathrm{EBV}^{+}$secondary lymphoma occurred later following CLL diagnosis ( 3.4 vs. 4.75 years, respectively, $p$ value $>0.05$ ) and in older patients (median age 66 vs. 74 years, respectively, $p$ value $>0.05$ ). There was no clear difference in prognosis (median survival 4.5 vs. 2.5 months, respectively, log-rank and Wilcoxon $p$ value $>0.05$; Fig. $2 \mathrm{a}$ ). The same observations were made when only the clonally related "true" RT cases were considered. Overall, three of four $\mathrm{EBV}^{+}$and seven of seven $\mathrm{EBV}^{-}$secondary lymphomas analyzed were clonally related to the CLL. Also in this series, there was a trend that $\mathrm{EBV}^{+}$ RT occurred later after CLL diagnosis (4.2 vs. 2.9 years, respectively, $p$ value $>0.05$ ) in older patients (median age 75 vs. 66 years respectively, $p$ value $>0.05$ ) and with a worse outcome (median 4 vs. 7.25 months, respectively, log-rank and Wilcoxon $p$ value $>0.05$; Fig. 2b).

None of the comparisons was significant, likely due to small sample size.

Table 1 Clinical features of 16 CLL cases with secondary B-cell lymphoma from UZ Leuven

\begin{tabular}{|c|c|c|c|c|c|c|c|c|c|}
\hline Case & Sex & $\begin{array}{l}\text { Age at Dx } \\
\text { CLL (years) }\end{array}$ & $\begin{array}{l}\text { Mutation } \\
\text { status CLL }\end{array}$ & CLL therapy & $\begin{array}{l}\text { Interval CLL/s BCL } \\
\text { (age at sec BCL), years }\end{array}$ & Diagnosis & $\begin{array}{l}\text { OS } \\
\text { (months) }\end{array}$ & State & ROD \\
\hline \multicolumn{10}{|c|}{$\mathrm{EBV}^{+}$secondary B-cell lymphoma } \\
\hline 1 & M & 72 & ND & Chl, F & $4.2(76)$ & DLBCL & 4.5 & $\mathrm{D}$ & Sepsis \\
\hline 2 & M & 70 & UNM & Chl, F & $5.25(75)$ & DLBCL & 1 & $\mathrm{D}$ & Sepsis \\
\hline 3 & $\mathrm{~F}$ & 65 & MUT & $\mathrm{F}, \mathrm{A}$ & $4.25(69)$ & DLBCL & 48 & $\mathrm{~A}$ & - \\
\hline 4 & M & 73 & ND & None & $1.3(74)$ & DLBCL & 4 & $\mathrm{D}$ & Progression \\
\hline 5 & M & 65 & MUT & Chl, F, A & $9(74)$ & DLBCL & 1 & $\mathrm{D}$ & Progression \\
\hline 6 & $\mathrm{~F}$ & 58 & MUT & Chl, FCR & $7.25(65)$ & $\mathrm{cHL}$ & 8 & $\mathrm{D}$ & Progression \\
\hline \multicolumn{10}{|c|}{ EBV $^{-}$secondary B-cell lymphoma } \\
\hline 7 & $\mathrm{~F}$ & 76 & ND & None & $0(76)$ & DLBCL & 11.75 & A & - \\
\hline 8 & $\mathrm{~F}$ & 72 & ND & Chl & $5.4(77)$ & DLBCL & 0.75 & A & - \\
\hline 9 & M & 63 & ND & FC, FCR & $8(71)$ & DLBCL & 13.5 & $\mathrm{D}$ & Progression \\
\hline 10 & M & 49 & MUT & $\mathrm{FC}, \mathrm{CHOP}$ & $6.2(55)$ & DLBCL & 1.5 & $\mathrm{D}$ & Progression \\
\hline 11 & $\mathrm{~F}$ & 65 & ND & F, FMD & $1.6(66)$ & DLBCL & 10 & $\mathrm{D}$ & Progression \\
\hline 12 & M & 38 & UNM & FCR, A, alloBMT & $3.9(42)$ & DLBCL & 9.5 & A & - \\
\hline 13 & $\mathrm{~F}$ & 57 & UNM & Chl, F, O & $8.8(66)$ & DLBCL & 3 & $\mathrm{D}$ & Progression \\
\hline 14 & M & 54 & UNM & F, A, alloBMT & $2.2(56)$ & DLBCL & 4.5 & $\mathrm{D}$ & Progression \\
\hline 15 & M & 65 & ND & Chl, FCR & $0.1(65)$ & DLBCL & 1.5 & $\mathrm{D}$ & Progression \\
\hline 16 & M & 75 & ND & Chl & $2.9(78)$ & DLBCL & 32.5 & $\mathrm{D}$ & CVA \\
\hline
\end{tabular}

$A$ alive; $C L L$ chronic lymphocytic leukemia; $c H L$ classic Hodgkin lymphoma; $C h l$ chlorambucil; $C V A$ cerebrovascular accident; $D$ dead; $D x$ diagnosis; $F$ female; $F$ fludarabine; $F C$ fludarabine and cyclophosphamide; $F C A$ fludarabine, cyclophosphamide, alemtuzumab; $F C R$ fludarabine, cyclophosphamide, rituximab; $F M D$ fludarabine, mitoxantrone, dexamethasone; $M U T$ mutated; $M$ male; $N A$ not available; $N D$ not determined; $O$ ofatumumab, sec $B C L$ secondary B-cell lymphoma; $U N M$ unmutated; $Y$ yes 
Table 2 Pathological features of 16 CLL cases with secondary B-cell lymphoma from UZ Leuven

\begin{tabular}{|c|c|c|c|c|c|c|c|c|c|c|c|c|c|c|}
\hline & CLL & Secondary B-cel & lymphoma & & & & & & & & & & & \\
\hline Case & $\begin{array}{l}\text { Related } \\
\text { aberrations }\end{array}$ & $\begin{array}{l}\text { Related to CLL } \\
\text { (by Ig PCR) }\end{array}$ & $\begin{array}{c}\text { EBER/LMP1/ } \\
\text { EBNA2/ } \\
\text { ZEBRA }\end{array}$ & $\mathrm{CD} 20$ & CD5 & $\mathrm{CD} 23$ & $\mathrm{CD} 10$ & BCL6 & MUM1 & BCL2 & Mib1 & CyD1 & TP53 & MYC \\
\hline \multicolumn{15}{|c|}{$\mathrm{EBV}^{+}$secondary B-cell lymphoma } \\
\hline 1 & $\operatorname{del}(11 q)$ & $\mathrm{Y}$ & $+/+/+/-$ & + & + & Part & - & - & + & Weak & $70 \%$ & - & $5 \%$ & $0 \%$ \\
\hline 2 & $\operatorname{del}(13 q)$ & $\mathrm{Y}$ & $+/ \mathrm{NR} /+/-$ & + & + & Part & - & - & + & + & $90 \%$ & - & $5 \%$ & $5 \%$ \\
\hline 3 & $\operatorname{del}(13 q)$ & $\mathrm{N}$ & $+/+/-/-$ & + & + & - & - & + & + & + & $75 \%$ & - & $50 \%$ & $10 \%$ \\
\hline 4 & ND & $\mathrm{Y}$ & $+/+/-/+$ & + & + & + & - & - & + & - & $80 \%$ & - & $5 \%$ & $10 \%$ \\
\hline 5 & $\begin{array}{l}\operatorname{del}(13 q) \\
\quad \operatorname{del}(17 p) \\
\operatorname{del}(13 q) \\
\operatorname{dem}(13 q)\end{array}$ & NA & $+/ \mathrm{NA}$ & + & NA & NA & - & Part & + & + & $85 \%$ & NA & $5 \%$ & NA \\
\hline 6 & ND & NA & + /NA/NA/- & - & NR & - & - & - & + & + & NA & NA & $90 \%$ & $\begin{array}{c}10 \% \\
\text { RS } \\
\text { cells }\end{array}$ \\
\hline \multicolumn{15}{|c|}{$\mathrm{EBV}^{-}$secondary B-cell lymphoma } \\
\hline 7 & ND & $\mathrm{Y}$ & - & + & Weak & Part & - & + & + & + & $80 \%$ & - & $5 \%$ & $10 \%$ \\
\hline 8 & ND & NA & - & + & - & - & - & - & NR & - & $70 \%$ & - & $5 \%$ & $5 \%$ \\
\hline 9 & $\begin{array}{l}\operatorname{del}(11 q) \\
\operatorname{del}(13)\end{array}$ & $\mathrm{Y}$ & - & + & + & Weak & - & - & - & - & $80 \%$ & - & $75 \%$ & $1 \%$ \\
\hline 10 & $\operatorname{del}(17 p)$ & $\mathrm{Y}$ & - & + & + & Part & - & - & - & + & $80 \%$ & - & $80 \%$ & $1 \%$ \\
\hline 11 & ND & $\mathrm{Y}$ & - & Weak & + & + & - & - & + & + & $90 \%$ & - & $1 \%$ & $5 \%$ \\
\hline 12 & $13 q$ & NA & - & + & + & + & - & Weak & + & + & $30 \%$ & - & $30 \%$ & $1 \%$ \\
\hline 13 & ND & $\mathrm{Y}$ & - & + & + & Part & - & - & + & + & $40 \%$ & - & $1 \%$ & $30 \%$ \\
\hline 14 & $+12, \operatorname{del}(17 \mathrm{p})$ & $\mathrm{Y}$ & - & + & - & Part & - & - & + & + & $80 \%$ & - & $60 \%$ & $25 \%$ \\
\hline 15 & ND & NA & - & + & + & Part & - & - & + & + & $80 \%$ & - & $80 \%$ & $30 \%$ \\
\hline 16 & +12 & $\mathrm{Y}$ & - & + & + & + & - & - & - & + & $60 \%$ & - & $1 \%$ & $10 \%$ \\
\hline
\end{tabular}

$C L L$ chronic lymphoid leukemia, EBER Epstein-Barr virus-encoded RNA, FISH fluorescent in situ hybridization, $N$ no, $N A$ not available, $N R$ not representative, $N D$ not detected, $R S$ cells Reed-Sternberg cells, $Y$ yes

Whereas the BCL2 protein was expressed in nearly all cases, high TP53 (50 vs. $33 \%$ ) and MYC (30 vs. $0 \%$ ) expression seemed more common in $\mathrm{EBV}^{-}$than $\mathrm{EBV}^{+} \mathrm{RT}$ (Table 2).

In the $\mathrm{EBV}^{+}$cases, the $\mathrm{EBER}^{+}$cells expressed an intermediate (LMP1+/EBNA2-, 2/4) or broad latency type (LMP1+/ EBNA2+, 2/4). Notably, in one case (no. 4), the lytic protein ZEBRA was expressed in a small fraction of tumor cells indicative of active lytic replication.

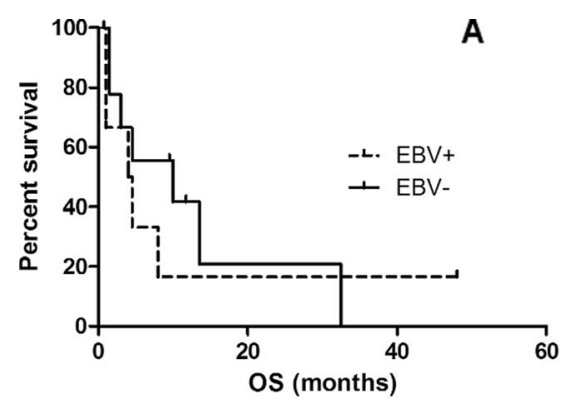

Fig. 2 Survival analysis of secondary B-cell lymphoma. Kaplan-Meier plot comparing overall survival of all EBV-positive $(n=6$, dashed line $)$ and EBV-negative ( $n=10$, full line) secondary B-cell lymphoma (a, log-

\section{DISCUSSION}

The Epstein-Barr virus (EBV) is an oncogenic human herpesvirus that has been associated with the development of lymphoproliferative disorders (LPD), in particular in immunocompromised individuals. It has been suggested that in some cases EBV may play a role in the transformation of indolent CLL to aggressive DLBCL, referred to as Richter

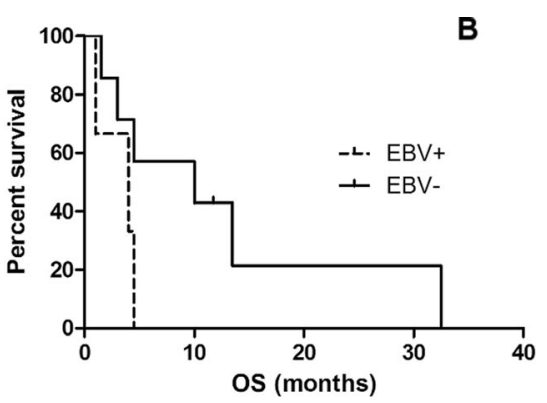

rank $p$ and Wilcoxon $p$ value $>0.05$ ) and of EBV-positive ( $n=3$, dashed line) and EBV-negative Richter transformation ( $n=7$, full line) (b, logrank $p$ and Wilcoxon $p$ value $>0.05$ ) in CLL patients 
transformation. According to current estimates, EBV is present in $6 \%$ of the DLBCL variant of RT [19]. In our case series, however, EBV was present in $38 \%(6 / 16)$ of DLBCL RT cases suggesting that the involvement of EBV in this disorder is underestimated. We therefore propose that newly diagnosed cases of RT are systematically screened for the presence of EBV by in situ hybridization, the most reliable method to determine the presence of EBV.

Interesting differences between the cases of with $\mathrm{EBV}^{+}$ secondary B-cell lymphoma were found.

Case 1 and 5 are classic cases of a fludarabine-associated EBV-driven LPD arising shortly after completion of therapy. Purine analogs, like fludarabine and cladribine, are highly effective treatments for low-grade B-cell neoplasms [20], but the accompanying long-lasting T-cell suppression, a major side effect, is related to the development of $\mathrm{EBV}^{+}$LPDs [21, 22]. Less clear is the situation for case 2 , who only received two doses of fludarabine and developed the LPD 34 months after. Intervals between completion of fludarabine and development of lymphoma were reported to be up to 1 year [21].

Case 3 developed a large B-cell lymphoma 2 months after completion of alemtuzumab monotherapy. Alemtuzumab targets CD52, present on nearly all $\mathrm{B}$ and T lymphocytes, monocytes, and natural killer (NK) cells, and is thus highly immunosuppressive. Prolonged T-cell deficiency induced by alemtuzumab is known to facilitate opportunistic infections and specifically to impair recovery of EBV-specific CD8+ T lymphocytes making the patient vulnerable for EBV-driven lymphoproliferation. Despite the large numbers of patients with chronic lymphocytic leukemia or T-prolymphocytic leukemia treated with alemtuzumab, the occurrence of $\mathrm{EBV}^{+}$ lymphoproliferative disease is very rare, especially during or following monotherapy [21, 23]. Whether there was a synergistic immunosuppressive effect with the fludarabine treatment this patient had received more than 4 years before is hard to define.

Case 4, with isolated RT of the brain, is unique for several reasons. Although asymptomatic secondary CNS infiltration by CLL has been shown at autopsy in 8 to $70 \%$ of the cases [24-26], symptomatic involvement of the CNS by CLL cells is a relatively rare event. Isolated RT of the brain is even more rare: only 13 cases have been described in detail so far [11, 24, $27,28]$, five of which had isolated leptomeningeal involvement, and eight demonstrated parenchymal involvement. Our patient had a history of different malignancies (prostate cancer, B-ALL, CLL, RT) but only received chemotherapy, including intrathecal methotrexate (MTX), for the B-ALL. MTX is frequently administered to patients with autoimmune diseases, especially rheumatoid arthritis, to suppress the hyper-immune state. Since the first case of lymphoma in a rheumatoid arthritis patient who received low-dose MTX was reported [20], MTXinduced immunosuppression has been linked to development of EBV-driven LPD [29]. However, the therapeutic or prophylactic administration of high-dose intrathecal MTX like in this case has - to our knowledge - not yet been associated with the development of $\mathrm{EBV}^{+} \mathrm{LPD}$ of the brain.

As evidenced by cases, 3 , and 6 , sequential monitoring of EBV titers proved useful to predict the development of EBVpositive LPD in our patients, similarly to what has been described for post-transplant LPD patients [30]. We suggest that follow-up of EBV titers of CLL patients should be performed when evolution to RT is suspected, particularly in CLL patients receiving T-cell-suppressing therapy. This hypothesis is supported by a recent report suggesting that EBV reactivation may in fact be an under-diagnosed event in severely immunosuppressed CLL [31].

In contrast to DLBCL RT, the Hodgkin lymphoma variant of RT is rare and commonly positive for EBV. As for our case (no. 6), a retrospective study showed that mixed cellularity Hodgkin lymphoma is the most common subtype [32].

\section{CONCLUSION}

Genetic studies have identified several lesions (abnormalities of TP53, MYC, CDKN2A, and IGH-mediated translocations) $[11,33,34]$ associated with RT pathogenesis, and currently a number of genetic biomarkers (NOTCH1 mutation, IGHV439) are used to identify CLL patients at risk of RT. Based on this small study, we propose EBV as an additional biomarker to predict RT development. Based on our observations, we suggest that $\mathrm{EBV}^{+} \mathrm{RT}$ may be more frequent than is currently assumed. Larger series are required to validate our hypotheses.

\section{Compliance with ethical standards}

Conflict of interest Julie Morscio, Emilie Bittoun, Nathalie Volders, Eveline Lurquin, Iwona Wlodarska, Olivier Gheysens, Peter Vandenberghe, Gregor Verhoef, Philippe Demaerel, Daan Dierickx, Xavier Sagaert, Ann Janssens, and Thomas Tousseyn declare that they have no conflict of interest.

Funding This study was funded by the K.U. Leuven (grant GOA/11/ 010 to IW and TT) and the FWO-Vlaanderen (G081411N to GV and TT). $\mathrm{XS}$ is FWO senior clinical investigator. TT is a postdoctoral researcher from Stichting tegen Kanker (2014-083).

Ethical approval This article does not contain any studies with human participants or animals performed by any of the authors. The use of human biopsy material in this study was approved by the Ethical Committee of the University Hospitals of Leuven (S-55498).

Open Access This article is distributed under the terms of the Creative Commons Attribution 4.0 International License (http:// creativecommons.org/licenses/by/4.0/), which permits use, duplication, adaptation, distribution and reproduction in any medium or format, as long as you give appropriate credit to the original author(s) and the source, provide a link to the Creative Commons license and indicate if changes were made. 


\section{References}

1. Jamroziak K, Tadmor T, Robak T, Polliack A. Richter syndrome in chronic lymphocytic leukemia: updates on biology, clinical features and therapy. Leukemia \& Lymphoma. 2015:1-10. Epub 2014/10/31.

2. Rossi D, Cerri M, Capello D, et al. (2008) Biological and clinical risk factors of chronic lymphocytic leukaemia transformation to Richter syndrome. Br J Haematol 142(2):202-215 Epub 2008/05/22

3. Rinaldi A, Mensah AA, Kwee I, et al. (2013) Promoter methylation patterns in Richter syndrome affect stem-cell maintenance and cell cycle regulation and differ from de novo diffuse large B-cell lymphoma. Br J Haematol 163(2):194-204 Epub 2013/08/22

4. Rossi D, Spina V, Forconi F, et al. (2012) Molecular history of Richter syndrome: origin from a cell already present at the time of chronic lymphocytic leukemia diagnosis. Int J Cancer J Int du cancer 130(12):3006-3010 Epub 2011/07/29

5. Trbusek M, Malcikova J (2013) TP53 aberrations in chronic lymphocytic leukemia. Adv Exp Med Biol 792:109-131 Epub 2013/ $09 / 10$

6. Dohner H, Fischer K, Bentz M, Hansen K, Benner A, Cabot G, et al. (1995) p53 gene deletion predicts for poor survival and nonresponse to therapy with purine analogs in chronic B-cell leukemias. Blood 85(6):1580-1589 Epub 1995/03/15

7. Rossi D, Rasi S, Spina V, et al. (2012) Different impact of NOTCH1 and SF3B1 mutations on the risk of chronic lymphocytic leukemia transformation to Richter syndrome. Br J Haematol 158(3):426429 Epub 2012/05/11

8. Rossi D, Spina V, Cerri M, et al. Stereotyped B-cell receptor is an independent risk factor of chronic lymphocytic leukemia transformation to Richter syndrome. Clin Cancer Res Off J Am Assoc Cancer Res 2009; 15(13):4415-4422. Epub 2009/06/11.

9. Rossi D, Gaidano G (2009) Richter syndrome: molecular insights and clinical perspectives. Hematol Oncol 27(1):1-10 Epub 2009/ $02 / 12$

10. Nakamura N, Abe M (2003) Richter syndrome in B-cell chronic lymphocytic leukemia. Pathol Int 53(4):195-203 Epub 2003/04/05

11. Chigrinova E, Rinaldi A, Kwee I, Rossi D, Rancoita PM, Strefford JC, et al. (2013) Two main genetic pathways lead to the transformation of chronic lymphocytic leukemia to Richter syndrome. Blood 122(15):2673-2682 Epub 2013/09/06

12. Ansell SM, Li CY, Lloyd RV, Phyliky RL (1999) Epstein-Barr virus infection in Richter's transformation. Am J Hematol 60(2):99-104 Epub 1999/02/03

13. Robertson LE, Pugh W, O’Brien S, et al. (1993) Richter's syndrome: a report on 39 patients. J Clin Oncol Off J Am Soc Clin Oncol 11(10):1985-1989 Epub 1993/10/01

14. Teramoto N, Gogolak P, Nagy N, et al. (2000) Epstein-Barr virusinfected B-chronic lymphocyte leukemia cells express the virally encoded nuclear proteins but they do not enter the cell cycle. J Hum Virol 3(3):125-136 Epub 2000/07/06

15. Stein H HN, Campo E. et al. Mature B-cell Neoplasms WHO Classification of Tumours of Haematopoietic and Lymphoid Tissues: Lyon: IARC; 2008. p. 179-267.

16. van Dongen JJ, Langerak AW, Bruggemann M, Evans PA, Hummel M, Lavender FL, et al. (2003) Design and standardization of PCR primers and protocols for detection of clonal immunoglobulin and T-cell receptor gene recombinations in suspect lymphoproliferations: report of the BIOMED-2 concerted action BMH4-CT98-3936. Leuk Off J Leuk Soc Am Leuk Res Fund UK 17(12):2257-2317 Epub 2003/12/13

17. Giudicelli V, Chaume D, Lefranc MP (2005) IMGT/GENE-DB: a comprehensive database for human and mouse immunoglobulin and $\mathrm{T}$ cell receptor genes. Nucleic Acids Res 33(Database issue): D256-D261 Epub 2004/12/21

18. Green MR, Monti S, Rodig SJ, Juszczynski P, Currie T, O’Donnell E, et al. (2010) Integrative analysis reveals selective 9p24.1 amplification, increased PD-1 ligand expression, and further induction via JAK2 in nodular sclerosing Hodgkin lymphoma and primary mediastinal large B-cell lymphoma. Blood 116(17):3268-3277 Epub 2010/07/16

19. Rossi D, Gaidano G (2013) Richter syndrome. In: Malek S (ed) Advances in chronic lymphocytic leukemia. Springer, New York, pp. 173-191

20. Ellman MH, Hurwitz H, Thomas C, Kozloff M (1991) Lymphoma developing in a patient with rheumatoid arthritis taking low dose weekly methotrexate. J Rheumatol 18(11):1741-1743 Epub 1991/ $11 / 01$

21. Shields DJ, Byrd JC, Abbondanzo SL, Lichy JH, Diehl LF, Aguilera NI (1997) Detection of Epstein-Barr virus in transformations of low-grade B-cell lymphomas after fludarabine treatment. Modern Pathol Official Journal of the United States and Canadian Academy of Pathology, Inc 10(11):1151-1159 Epub 1997/12/05

22. Abruzzo LV, Rosales CM, Medeiros LJ, Vega F, Luthra R, Manning JT, et al. (2002) Epstein-Barr virus-positive B-cell lymphoproliferative disorders arising in immunodeficient patients previously treated with fludarabine for low-grade B-cell neoplasms. Am J Surg Pathol 26(5):630-636 Epub 2002/04/30

23. Ghobrial IM, Otteman LA, White WL (2003) An EBV-positive lymphoproliferative disorder after therapy with alemtuzumab. N Engl J Med 349(26):2570-2572 discussion 2570-2. Epub 2003/12/26.

24. Robak T, Gora-Tybor J, Tybor K, Jamroziak K, Robak P, Kordek R, et al. (2004) Richter's syndrome in the brain first manifested as an ischaemic stroke. Leuk Lymphoma 45(6):1261-1267 Epub 2004/09/14

25. Cramer SC, Glaspy JA, Efird JT, Louis DN (1996) Chronic lymphocytic leukemia and the central nervous system: a clinical and pathological study. Neurology 46(1):19-25 Epub 1996/01/01

26. Jellinger K, Radiaszkiewicz T (1976) Involvement of the central nervous system in malignant lymphomas. Virchows Arch A, Pathol Anat Histol 370(4):345-362 Epub 1976/07/21

27. Ghofrani M, Tantiwongkosi B, Smith AS, Wasdahl DA (2007) Richter transformation of chronic lymphocytic leukemia presenting as a dural-based non-Hodgkin lymphoma mass. AJNR Am J Neuroradiol 28(2):318-320 Epub 2007/02/14

28. Ishida F, Nakazawa H, Takezawa Y, Matsuda K, Asano N, Sano K, et al. (2013) Richter transformation in the brain from chronic lymphocytic leukemia. J Clin Exp Hematopathol JCEH 53(2):157-160 Epub 2013/09/03

29. Mariette X, Cazals-Hatem D, Warszawki J, Liote F, Balandraud N, Sibilia J, et al. (2002) Lymphomas in rheumatoid arthritis patients treated with methotrexate: a 3-year prospective study in France. Blood 99(11):3909-3915 Epub 2002/05/16

30. Ohga S, Kubo E, Nomura A, Takada H, Suga N, Ishii E, et al. (2001) Quantitative monitoring of circulating Epstein-Barr virus DNA for predicting the development of posttransplantation lymphoproliferative disease. Int J Hematol 73(3):323-326 Epub 2001/05/10

31. Rath J, Geisler C, Christiansen CB, Hastrup N, Madsen HO, Andersen MK, et al. (2008) Epstein-Barr virus reactivation is a potentially severe complication in chronic lymphocytic leukemia patients with poor prognostic biological markers and fludarabine refractory disease. Haematologica 93(9):1424-1426 Epub 2008/07/22

32. Bockorny B, Codreanu I, Dasanu CA (2012) Hodgkin lymphoma as Richter transformation in chronic lymphocytic leukaemia: a retrospective analysis of world literature. Br J Haematol 156(1):50-66 Epub 2011/10/25

33. De Keersmaecker K, Michaux L, Bosly A, Graux C, Ferreiro JF, Vandenberghe P, et al. (2012) Rearrangement of NOTCH1 or BCL3 can independently trigger progression of CLL. Blood 119(16): 3864-3866 Epub 2012/04/21

34. Podgornik H, Pretnar J, Skopec B, Andoljsek D, Cernelc P (2014) Concurrent rearrangements of BCL2, BCL3, and BCL11A genes in atypical chronic lymphocytic leukemia. Hematology 19(1):45-48 Epub 2013/02/26 